\title{
Transmit Beamforming in Multiple-Antenna Systems With Finite Rate Feedback: A VQ-Based Approach
}

\author{
June Chul Roh, Member, IEEE, and Bhaskar D. Rao, Fellow, IEEE
}

\begin{abstract}
This paper investigates quantization methods for feeding back the channel information through a low-rate feedback channel in the context of multiple-input single-output (MISO) systems. We propose a new quantizer design criterion for capacity maximization and develop the corresponding iterative vector quantization (VQ) design algorithm. The criterion is based on maximizing the mean-squared weighted inner product (MSwIP) between the optimum and the quantized beamforming vector. The performance of systems with quantized beamforming is analyzed for the independent fading case. This requires finding the density of the squared inner product between the optimum and the quantized beamforming vector, which is obtained by considering a simple approximation of the quantization cell. The approximate density function is used to lower-bound the capacity loss due to quantization, the outage probability, and the bit error probability. The resulting expressions provide insight into the dependence of the performance of transmit beamforming MISO systems on the number of transmit antennas and feedback rate. Computer simulations support the analytical results and indicate that the lower bounds are quite tight.
\end{abstract}

Index Terms-Bit error probability, channel capacity, channel state information, multiple antennas, transmit beamforming, outage probability, vector quantization (VQ).

\section{INTRODUCTION}

C OMMUNICATION systems using multiple antennas have attracted considerable attention because of the potential improvements in transmission rate and/or diversity gain. The performance achievable using multiple antennas depends on the nature of channel state information (CSI) available at the transmitter and at the receiver. We assume perfect CSI at the receiver and focus on the CSI at the transmitter (CSIT). Two extreme but common CSIT assumptions are complete CSIT where perfect channel information is known to the transmitter (e.g., [1], [2]), and no CSIT (e.g., [1]). The former case, complete CSIT, provides a higher capacity link than the latter in the single-user case [3], and has other benefits such as lower complexity receivers [4] and better system throughput in a multiuser environment [5]. However, the assumption that the transmitter has per-

Manuscript received August 17, 2004; revised June 25, 2005. This work was supported by the University of California Discovery Grant core02-10109 sponsored by Ericsson. The material in this paper was presented in part at the 38th Asilomar Conference on Signals, Systems, and Computers, Pacific Grove, CA, November 2004.

J. C. Roh was with the Department of Electrical and Computer Engineering, the University of California, San Diego, La Jolla, CA 92093-0407 USA. He is now with Texas Instruments, Inc., Sunnyvale, CA 94089 USA (e-mail: jroh@ti.com).

B. D. Rao is with the Department of Electrical and Computer Engineering, the University of California, San Diego, La Jolla, CA 92093-0407 USA (e-mail: brao@ece.ucsd.edu).

Communicated by V. V. Vaishampayan, Associate Editor At Large.

Digital Object Identifier 10.1109/TIT.2005.864426 fect knowledge of multidimensional channel is unrealistic; as in many practical systems, the channel information is provided to the transmitter through a finite-rate feedback channel. In this paper, we focus our attention on multiple-input single-output (MISO) systems and on the problem of feeding back the beamforming vector through a finite-rate feedback channel. Many interesting methods for feeding back beamforming information have been suggested [6] and evaluated mainly through simulations. Only recently, more systematic methods to analytically quantify the performance of finite-rate feedback systems have begun to appear [3], [4], [7]-[9].

For a MISO channel, the channel information for feedback is a complex unit-norm beamforming vector. In this paper, as in [3], we treat the problem as a vector quantization (VQ) problem, a subject that has received much attention in source coding [10]-[12]. For a given number of bits, full search VQ techniques are known to result in optimal quantizers. In addition, well-established algorithms for designing these quantizers are available making them very easy to use. However, the often employed mean-squared error (MSE) criterion in source coding, though convenient for quantization design, is not very suitable in our context because the more appropriate criteria for effective communication is channel capacity or signal-to-noise ratio (SNR). Maximizing mutual information could be a good design criterion (this was studied in [13] for multiple-input multiple-output (MIMO) channels). However, unfortunately, using it directly does not lead to a well-behaved iterative quantizer design algorithm in a sense that the algorithm does not guarantee an improvement in the design objective at every iteration (details will be discussed in Section III). This requires consideration and development of an appropriate design criterion that leads to effective quantizer design. We propose a new design criterion, namely, maximizing the mean-squared weighted inner product (MSwIP) which is directly related to channel capacity. The new design criterion does lead to a Lloyd-type VQ design algorithm that has a closed-form centroid solution thereby guaranteeing a better quantizer at every iteration. The approach is a generalization of the method suggested in [3].

We then analyze the performance of quantized beamforming systems. Our work continues along the analytical vein established in [3], [4], [7] and attempts to further the analytical understanding of quantized beamformers. Many interesting results on multiple-antenna systems with partial side information for both point-to-point links and broadcast systems have been developed in [3]. One aspect of this work involves performance limits of quantized beamformers. In our work, we conduct a rigorous analysis of the quantizer for the independent and identically distributed (i.i.d.) Rayleigh-fading channel. We derive an 
expression for the lower bound on the capacity loss. For a fixed number of transmit antennas, it is found to be quite tight as the number of quantization levels or feedback bits increases. Our results improve on the analysis results in [3] in several ways: the expressions are more precise, they are not based on an MSE criterion, and they are not asymptotic in the number of antennas.

More recently, improved results on the performance of finite-rate feedback systems has been developed in [4], [7]. For beamforming codebook construction, they consider a min-max criterion, i.e., minimizing the maximum inner product among all possible pairs of beamforming vectors. The connection between quantized beamformers and Grassmanian line packing was established to solve and expose the structure in the codebook. Even though our VQ-based design procedure does not provide an explicit form for the code points, this is of minimal inconvenience given the generality of the design methodology and flexibility of the framework. The design methods readily apply to a variety of statistical environments, and the framework is rich enough to support interesting complexity-to-performance tradeoffs. With the MSwIP design procedure, one can design the optimum quantizer for any number of transmit antennas, any number of quantization bits, and spatial correlation.

Analytical results were also developed in [4] to characterize the performance of optimally quantized beamformers. For instance, a universal lower bound was derived for the outage probability for any finite set of optimum beamformers. In [7], new results on Grassmanian line packing are developed and used to evaluate the optimality of quantized beamformers. Though our analytical approach has points of commonality with those of [4], [7], it is quite different in flavor and offers an interesting alternative. In particular, in our approach the squared inner product between the optimum and the quantized beamforming vector is statistically characterized and used in deriving the capacity loss resulting from finite-rate feedback of channel information. The approach taken leads to more precision in the analysis and in our opinion is also a simpler analytical approach. The power of the approach is further demonstrated by extending the analytical results to derive the outage probability and the bit error probability for a finite-rate feedback MISO system. The framework for codebook construction and performance analysis can be extended to MIMO systems. Though the extensions are not completely general, the framework for codebook construction has been extended to the equal power case and a limited performance analysis provided for the i.i.d. flat-fading channel scenario [14], [15]. Since the submission of this paper, additional work on the design and analysis of codebooks for the correlated channel case have appeared and the interested reader is referred to [16], [17] for more details.

This paper is organized as follows. Section II describes the system model and assumptions. The quantizer design for lowrate feedback of the beamforming vector in MISO systems is presented in Section III. In Section IV, the density function of the squared inner product is derived and then it is used to analyze the capacity loss due to quantization of beamforming vector. In Sections V and VI, the outage probability and the bit error probability for systems with quantized beamforming are analyzed, respectively, by directly applying the distributions derived in Section IV.
We use the following notations. $A^{\dagger}$ indicates the conjugate transpose of matrix $A$. The inner product between two vectors is defined as $\langle u, v\rangle=u^{\dagger} v$ and the 2-norm of vector $v$ is denoted by $\|v\|=\langle v, v\rangle^{1 / 2} . E[\cdot]$ represents the expectation operator, and $\tilde{\mathcal{N}}(\mu, \Sigma)$ and $\mathcal{N}(\mu, \Sigma)$ are, respectively, the proper complex and the real Gaussian random vector with mean $\mu$ and covariance $\Sigma$. Uniform distribution over a set $S$ is denoted by $\mathcal{U}(S)$. The function $\log (\cdot)$ is the natural logarithm.

\section{SYSTEM MODEL}

We consider a multiple-antenna system with $t$ antennas at the transmitter and a single antenna at the receiver. Assuming flat fading, the multiple-antenna channel is modeled by the channel vector $h \in \mathbb{C}^{t}$. That is, the channel input $x \in \mathbb{C}^{t}$ and the channel output $y \in \mathbb{C}$ have the following relationship:

$$
y=h^{\dagger} x+\eta
$$

where $\eta$ is the additive white Gaussian noise distributed by $\tilde{\mathcal{N}}(0,1)$. The average transmit power is denoted by $P_{T}$, i.e., $E\left[x^{\dagger} x\right]=P_{T}$. The channel vector will be also written in terms of its magnitude and direction as $h=\alpha v$, where $\alpha=\|h\|$ and $v=h /\|h\| \cdot \cdot^{1}$

It is well known that, with perfect CSIT, transmit beamforming along $v$ is the optimum choice for maximizing the received SNR and also the mutual information. In this paper, we will consider quantization of the unit-norm vector for feeding back to the transmitter through a feedback channel. The capacity of the feedback channel is assumed to be finite and limited to $B$ bits per channel update. For feedback purposes, a quantization codebook $\mathcal{C}=\left\{\hat{v}_{1}, \ldots, \hat{v}_{N}\right\}$, where $\left\|\hat{v}_{i}\right\|=1$ and $N=2^{B}$, is assumed known to both the receiver and the transmitter (designing the codebook will be dealt with in Section III). Based on the channel information, the receiver selects the best beamforming vector, say $\hat{v}$, from the codebook and sends the corresponding index of the selected beamforming vector to the transmitter. In practice, the error in the feedback channel and the delay resulting from finite-rate channel update impact the overall system performance. However, this paper assumes feedback with no error and no delay, and focuses on the effect of quantizing the channel information with a finite number of bits.

At the transmitter, $\hat{v}$ is employed as the transmit beamforming $\operatorname{vector}(\|\hat{v}\|=1)$. That is, an information-bearing symbol $s \in \mathbb{C}$ is transmitted through multiple antennas as $x=\hat{v} s$, resulting in the received signal

$$
y=\alpha\langle v, \hat{v}\rangle s+\eta
$$

where $E\left[|s|^{2}\right]=P_{T}$. In this setting, the received SNR is simply given by

$$
\mathrm{SNR}=\alpha^{2}|\langle v, \hat{v}\rangle|^{2} P_{T}
$$

and the mutual information between $s$ and $y$ for given $h$ and $\hat{v}$ is given by

$$
I(h, \hat{v})=\log \left(1+\alpha^{2}|\langle v, \hat{v}\rangle|^{2} P_{T}\right)
$$

${ }^{1}$ Though not explicitly indicated for notational simplicity, note that throughout the paper $\alpha$ and $v$ (also $\hat{v}$ later) are functions of $h$. 
in nats per channel use, where it is assumed that $s \sim \tilde{\mathcal{N}}\left(0, P_{T}\right)$. It is obvious that $I(h, \hat{v})$ with finite $B$ is less than the mutual information with perfect feedback (with $B=\infty$ )

$$
I(h, v)=\log \left(1+\alpha^{2} P_{T}\right)
$$

since $|\langle v, \hat{v}\rangle|^{2}$ is always less than one.

Let us define the capacity loss due to quantization of beamforming vector as the difference between the ergodic capacity with perfect and quantized feedback, that is,

$$
\begin{aligned}
C_{L} & =E[I(h, v)]-E[I(h, \hat{v})] \\
& =E\left[I_{L}(h, \hat{v})\right]
\end{aligned}
$$

where $E[I(h, v)]$ and $E[I(h, \hat{v})]$ are the ergodic capacities when the transmitter uses the optimum and the quantized beamforming vector, respectively; and

$$
I_{L}(h, \hat{v})=I(h, v)-I(h, \hat{v}) .
$$

With a little manipulation, it can be rewritten as

$$
I_{L}(h, \hat{v})=-\log \left(1-\frac{\alpha^{2} P_{T}}{1+\alpha^{2} P_{T}}\left(1-|\langle v, \hat{v}\rangle|^{2}\right)\right) .
$$

\section{QUANTIZATION OF RANDOM BEAMFORMING VeCTOR}

In this section, we develop a general VQ design method for the quantization of random beamforming vector.

\section{A. The MSwIP Criterion and VQ Design Algorithm}

For designing the beamforming codebook, a good design criterion could be to maximize the expected mutual information $E[I(h, \hat{v})]$ or, equivalently, to minimize the capacity loss defined in (6). However, unfortunately using it directly leads to difficulty with the centroid computation, an essential step in VQ design (see, e.g., [3], [13]). The difficulty with the approach is that generally there is no analytical expression for the optimum code vector as a function of a given partition region in the channel space. ${ }^{2}$ This necessitates approximation to the solution to the optimization problem as in [13]. Thus, the resulting iterative design algorithm does not necessarily maximize the original design criterion, which in turn makes it hard to guarantee the optimality of the resulting codebook.

The nature of the approximation becomes important, and in this paper we consider the following approximation to the capacity loss: When $|\langle v, \hat{v}\rangle|$ is close to one (which is valid when $N$ is reasonably large) $o r$ when $P_{T} \ll 1$, (7) can be approximated using $-\log (1-x) \simeq x$ for small $x$ as

$$
I_{L}(h, \hat{v}) \simeq \tilde{\alpha}^{2}\left(1-|\langle v, \hat{v}\rangle|^{2}\right)
$$

where

$$
\tilde{\alpha}=\sqrt{\frac{\alpha^{2} P_{T}}{1+\alpha^{2} P_{T}}} .
$$

\footnotetext{
${ }^{2}$ This is called the centroid condition in quantizer design.
}

This results in

$$
C_{L} \simeq E\left[\tilde{\alpha}^{2}\left(1-|\langle v, \hat{v}\rangle|^{2}\right)\right]
$$

which will be the basis of the quantizer design.

New Design Criterion: Design a quantizer $\mathcal{Q}$ (mathematically, $\mathcal{Q}: \mathbb{C}^{t} \rightarrow \mathcal{C}$ ) to maximize the $M S w I P$

$$
\max _{\mathcal{Q}(\cdot)} E|\langle\tilde{\alpha} v, \mathcal{Q}(h)\rangle|^{2}
$$

where $\hat{v}=\mathcal{Q}(h)$ is the quantized beamforming vector $(\|\hat{v}\|=$ 1 ) from codebook $\mathcal{C}$. This VQ design criterion will be called the MSwIP criterion.

One of the virtues of the MSwIP criterion is that it does lead to a closed-form VQ design algorithm (modified Lloyd algorithm) that has monotonic convergence property. ${ }^{3}$ The original Lloyd algorithm is based on two conditions: i) optimum encoder (partition regions) for a fixed decoder (code vectors), and ii) optimum decoder for a fixed encoder [10], [12]. They are also called the nearest neighborhood condition (NNC) and the centroid condition (CC), respectively. The same approach is used here for designing the quantizer.

\section{New Design Algorithm:}

1. $N N C$ : For given code vectors $\left\{\hat{v}_{i} ; i=1, \ldots, N\right\}$, the optimum partition cells satisfy

$$
\mathcal{R}_{i}=\left\{h \in \mathbb{C}^{t}:\left|\left\langle\tilde{\alpha} v, \hat{v}_{i}\right\rangle\right| \geq\left|\left\langle\tilde{\alpha} v, \hat{v}_{j}\right\rangle\right|, \forall j \neq i\right\}
$$

for $i=1, \ldots, N$, where $\mathcal{R}_{i}$ is the partition cell (Voronoi region) for the $i$ th code vector $\hat{v}_{i}$.

2. $C C$ : For a given partition $\left\{\mathcal{R}_{i} ; i=1, \ldots, N\right\}$, the optimum code vectors satisfy

$$
\hat{v}_{i}=\arg \max _{\hat{v} \in \mathcal{R}_{i},\|\hat{v}\|=1} E\left[|\langle\tilde{\alpha} v, \hat{v}\rangle|^{2} \mid h \in \mathcal{R}_{i}\right]
$$

for $i=1, \ldots, N$. Since

$$
E\left[|\langle\tilde{\alpha} v, \hat{v}\rangle|^{2} \mid h \in \mathcal{R}_{i}\right]=\hat{v}^{\dagger} E\left[\tilde{\alpha}^{2} v v^{\dagger} \mid h \in \mathcal{R}_{i}\right] \hat{v}
$$

the solution for the above optimization problem is

$$
\hat{v}_{i}=\text { (principal eigenvector) of } E\left[\tilde{\alpha}^{2} v v^{\dagger} \mid h \in \mathcal{R}_{i}\right] .
$$

The above two conditions are iterated until the MSwIP $E|\langle\alpha \tilde{\alpha} v, \mathcal{Q}(h)\rangle|^{2}$ converges. In practice, a quantizer is designed using a sufficiently large number of training samples (channel realizations). In that case, the statistical correlation matrix in (12) is estimated with an experimental expectation.

Beamforming Vector Selection (Encoding): For a given codebook $\mathcal{C}=\left\{\hat{v}_{1}, \ldots, \hat{v}_{N}\right\}$, the receiver encodes as follows: ${ }^{4}$

$$
\hat{v}=\mathcal{Q}(v)=\arg \max _{\hat{v}_{i} \in \mathcal{C}}\left|\left\langle v, \hat{v}_{i}\right\rangle\right| .
$$

This is the optimum encoding scheme that maximizes the received SNR and also the mutual information. By the encoding

\footnotetext{
${ }^{3}$ Monotonic convergence means here that an improved design is guaranteed at every iteration.

${ }^{4}$ The encoding process in beamforming vector selection is denoted by $\mathcal{Q}(v)$ since it only depends on $v$, while in designing a codebook in (9) the encoding is also dependent on $\alpha$, hence, it is denoted by $\mathcal{Q}(h)$.
} 
scheme, the unit-norm sphere $\mathcal{S}_{t}=\left\{u \in \mathbb{C}^{t}:\|u\|=1\right\}$, where random vector $v$ lies, is partitioned into $\left\{\overline{\mathcal{R}}_{i} ; i=1, \ldots, N\right\}$, where

$$
\overline{\mathcal{R}}_{i}=\left\{v \in \mathcal{S}_{t}:\left|\left\langle v, \hat{v}_{i}\right\rangle\right| \geq\left|\left\langle v, \hat{v}_{j}\right\rangle\right|, \forall j \neq i\right\}
$$

The encoding scheme (13) can be restated simply as $\mathcal{Q}(v)=\hat{v}_{i}$ if $v \in \overline{\mathcal{R}}_{i}$.

\section{B. Two Related Design Methods}

The MSwIP design method is general and can be applied even for spatially correlated channels. A drawback of the MSwIP criterion is that the quantizer is optimized for a particular SNR (or $P_{T}$ ). As a result, we may need more than one codebooks, especially if the system has multiple operating SNR points. ${ }^{5}$ Therefore, it would be interesting to find other design methods that do not depend on $P_{T}$.

As the SNR increases $\left(P_{T} \rightarrow \infty\right), \tilde{\alpha} \rightarrow 1$; hence, the MSwIP criterion (9) reduces to

$$
\max _{\mathcal{Q}(\cdot)} E|\langle v, \mathcal{Q}(v)\rangle|^{2}
$$

where $\hat{v}=\mathcal{Q}(v)$ is the quantized beamforming vector $(\|\hat{v}\|=$ $1)$. This design criterion will be called the MSIP criterion. One can see that this criterion is based only on $v$ and is independent of $\alpha$ and $P_{T}$. A design algorithm corresponding to this criterion is obtained by setting $\tilde{\alpha}=1$ in the NNC and the CC for the MSwIP criterion. It would be instructive to compare this criterion with the MSE criterion, which is quite common in source coding study and also implied in rate-distortion-based analysis. Since $v$ and $\hat{v}$ are both unit norm, in the MSE criterion, $\min E\|v-\hat{v}\|^{2}$ is equivalent to $\max E[\operatorname{Re}\langle v, \hat{v}\rangle]$. Thus, we can see that the MSE criterion maximizes only the real part of the inner product; therefore, the two criteria are different ${ }^{6}$ and the MSIP criterion is a better criterion in this context.

Another connection of the MSwIP criterion can be found by considering the other extreme case. When $P_{T} \ll 1$ (in the low-SNR region), $\tilde{\alpha} \simeq \sqrt{P_{T}} \alpha$; hence, the original criterion (9) becomes

$$
\max _{\mathcal{Q}(\cdot)} E|\langle h, \mathcal{Q}(h)\rangle|^{2}
$$

This design criterion is now based on the unnormalized channel vector $h$ instead of $v$. The design algorithm can be obtained by replacing $\tilde{\alpha} v$ in the $\mathrm{NNC}$ and the CC for the MSwIP criterion with $h$. This VQ design method was proposed and studied in [3]. Note that the design objective here also can be said to be maximizing the expectation of the received SNR (3).

It should be pointed out that when the channel has i.i.d. entries, i.e., $h \sim \tilde{\mathcal{N}}\left(0, I_{t}\right)$, the three design methods are equivalent, all maximizing $E[\mathrm{SNR}]$ or $E|\langle v, \hat{v}\rangle|^{2}$. Therefore, one can use the MSIP method. This follows from the observation that for a random channel $h \sim \tilde{\mathcal{N}}\left(0, I_{t}\right), v=h /\|h\|$ is independent

\footnotetext{
${ }^{5}$ The design can be easily modified to accommodate SNR with a known distribution, but we do not consider this option in the paper.

${ }^{6}$ Note that the two criteria are the same for the real vector case, but not for the complex vector case.
}

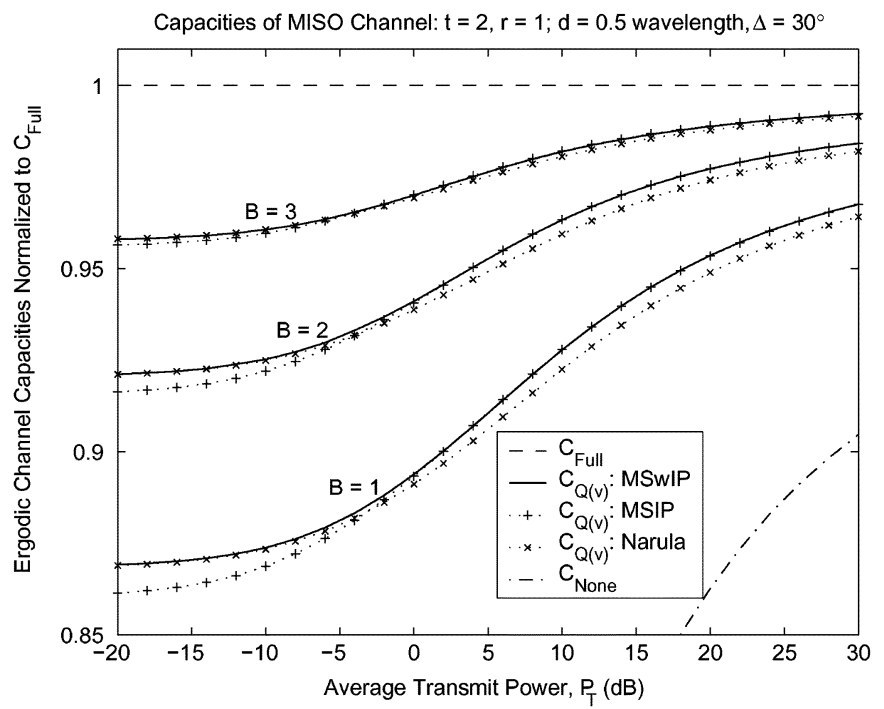

Fig. 1. Ergodic capacities of correlated MISO channels with quantized beamforming for the different quantizer design methods $(t=2$ and $B=1,2,3)$. Capacities are normalized to the capacity with complete CSIT $\left(C_{\text {Full }}\right) . C_{\text {None }}$ represents the capacity with no $\operatorname{CSIT}(B=0)$.

of $\alpha=\|h\|$ and uniformly distributed over the unit-norm sphere $\mathcal{S}_{t}$. However, for general spatially correlated channels, the three criteria are different since now $\alpha$ and $v$ are not independent.

\section{Design Examples and Discussion}

With the MSwIP design algorithm, we can obtain an optimum codebook for any number of transmit antennas and any number of codebook size. The performances of quantizer codebooks designed with the MSwIP design methods described in Sections III-A and III-B are compared in Fig. 1 in terms of the ergodic channel capacity, which is given by

$$
C_{\mathcal{Q}(v)}=E_{h}\left[\log \left(1+\alpha^{2}|\langle v, \mathcal{Q}(v)\rangle|^{2} P_{T}\right)\right] .
$$

For ease of comparison, all the capacities were normalized with respect to that of the complete CSIT $\left(C_{\text {Full }}\right)$. The channel is spatially correlated with the correlation model in [18]: A linear antenna array is simulated with antenna spacing of half wavelength and uniform angular spread in $\left[-30^{\circ}, 30^{\circ}\right]$. As expected, it turns out that in high-SNR region the MSIP method performs better than unnormalized channel vector quantization method of [3], and in low-SNR region the reverse relation holds. Moreover, the original MSwIP method always performs better than or equal to any of the other two methods over the entire SNR range. The performance difference decreases as $B$ increases, and generally the degree of performance improvement via the MSwIP method depends on the correlation parameters. As noted before, the MSwIP codebook is designed for each SNR point and the transmitter needs to know the operating SNR of the system $a$ priori. This can be implemented in most practical systems by selecting a set of SNR points and maintaining a particular SNR point over time using some form of power control mechanism. A simpler way with a minor performance loss is to partition the SNR region into two and use the codebook from the MSIP method in the high-SNR region and the unnormalized channel vector quantization method in the low-SNR region. 


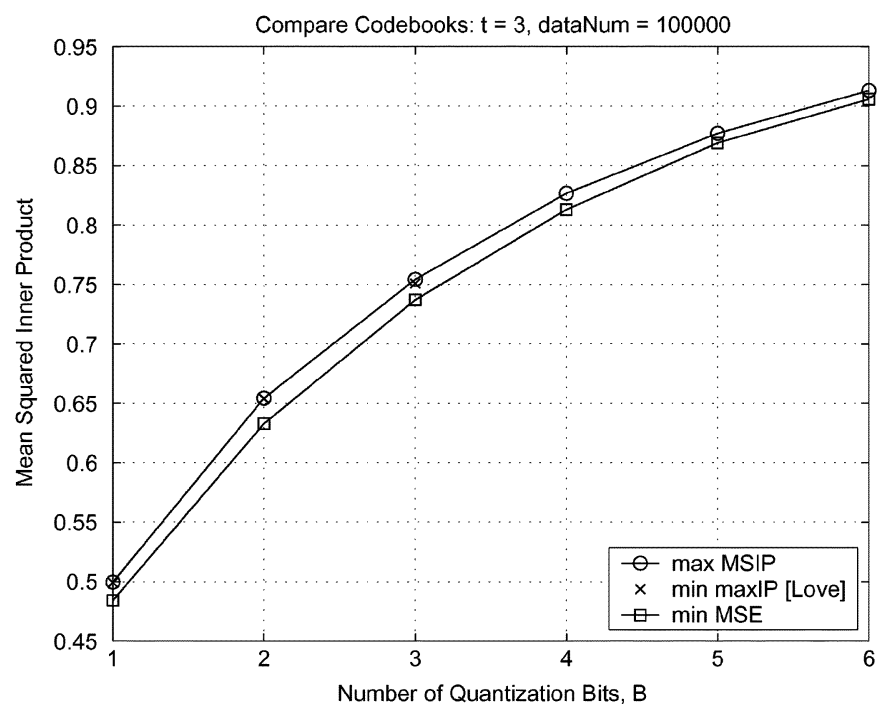

Fig. 2. Examples of quantizer design with the MSIP criterion and comparison with two other design criteria $(t=3$ and $B=1,2, \ldots, 6)$.

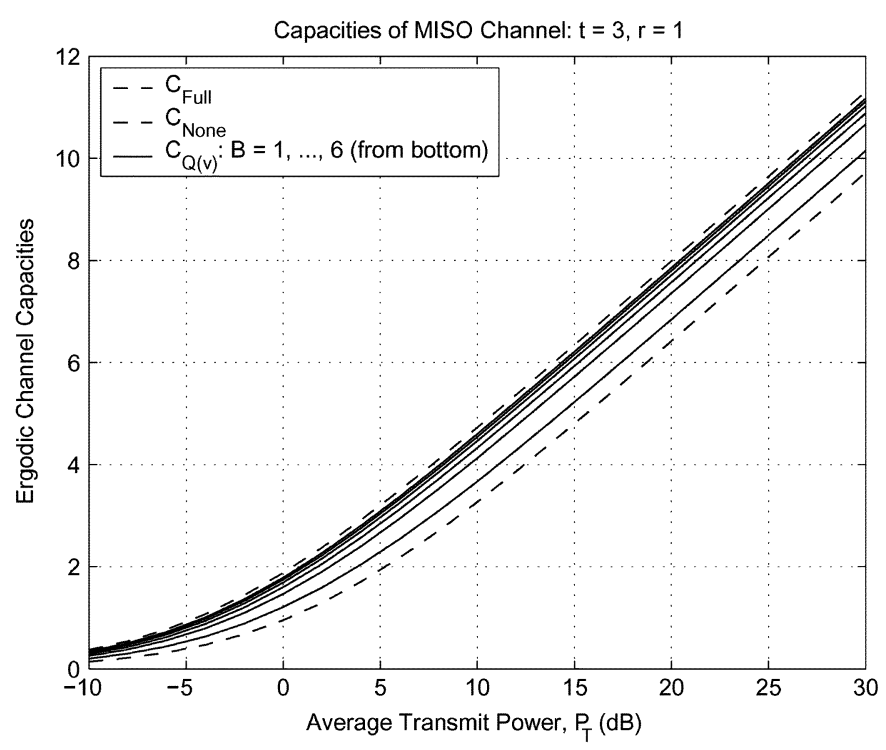

Fig. 3. Ergodic capacities of MISO channels with quantized beamforming in bits per channel use $(t=3$ and $B=1,2, \ldots, 6)$.

Design examples for the i.i.d. channel $h \sim \tilde{\mathcal{N}}\left(0, I_{t}\right)$ are shown in Figs. 2 and 3 for $t=3$ and various $B$. In Fig. 2, note that when $B=\infty$ the MSIP $E|\langle v, \hat{v}\rangle|^{2}=1$. For comparison, two other design methods are considered: i) $\min \max _{i \neq j}\left|\left\langle\hat{v}_{i}, \hat{v}_{j}\right\rangle\right|$; and ii) $\min E\|v-\hat{v}\|^{2}$ (MSE criterion). The first method has been studied in [4], [7]. Although it can be another reasonable design criterion, there is no VQ codebook design algorithm for arbitrary dimension and codebook size. The codebooks compared in the figure are from [7], where codebooks only for a low number of quantization bits $(B=1,2,3)$ are tabulated. Furthermore, the MSIP (also MSwIP) design approach is useful irrespective of the correlation structure of channel, while method i) is only good for the i.i.d. channel. The results show that the MSIP method performance is similar to that of method i), and performs better than the MSE method of ii). The ergodic capacities of MISO systems with quantized beamforming (using codebooks designed with the MSIP criterion) are shown in Fig. 3 when $t=3$ and $B=1, \ldots, 6$. For comparison, the capacities for the two extreme cases of complete $\operatorname{CSIT}\left(C_{\mathrm{Full}}\right)$ and no CSIT $\left(C_{\text {None }}\right)$ are added in the figure. The comparative results for the two competing methods in terms of MSIP also hold for the capacity measure (results are not shown).

\section{CAPACITY LosS WITH QUANTIZED FEEDBACK}

In this section, we will quantify the effect of quantization of the beamforming vector with a finite number of bits on the channel capacity for the i.i.d. MISO channel. With a given number of feedback (quantization) bits, we want to know how close we can approach to the performance of the complete feedback. The capacity loss defined in (6) will be analyzed in terms of $t$ and $B$. The capacity loss is repeated here as follows:

$$
\begin{aligned}
C_{L} & =E_{h}\left[I_{L}(h, \mathcal{Q}(v))\right] \\
& =E_{h}\left[-\log \left(1-\frac{\alpha^{2} P_{T}}{1+\alpha^{2} P_{T}}\left(1-|\langle v, \mathcal{Q}(v)\rangle|^{2}\right)\right)\right] .
\end{aligned}
$$

Since $\mathcal{Q}(v)=\hat{v}_{i} \forall h \in \mathcal{R}_{i}$ from (13), the capacity loss can be expressed as

$$
\begin{aligned}
C_{L}= & \sum_{i=1}^{N} P\left(h \in \mathcal{R}_{i}\right) E_{h \in \mathcal{R}_{i}}\left[I_{L}\left(h, \hat{v}_{i}\right)\right] \\
= & \sum_{i=1}^{N} P\left(h \in \mathcal{R}_{i}\right) \\
& \cdot E_{h \in \mathcal{R}_{i}}\left[-\log \left(1-\frac{\alpha^{2} P_{T}}{1+\alpha^{2} P_{T}}\left(1-\left|\left\langle v, \hat{v}_{i}\right\rangle\right|^{2}\right)\right)\right] .
\end{aligned}
$$

\section{A. Approximate Density Function of $|\langle v, \mathcal{Q}(v)\rangle|^{2}$}

In (18), note that $h \in \mathcal{R}_{i}$ implies $v \in \overline{\mathcal{R}}_{i}$. In order to calculate (18), we need to obtain the conditional density of the inner product $\gamma_{i}:=\left|\left\langle v, \hat{v}_{i}\right\rangle\right|^{2}$ given $v \in \overline{\mathcal{R}}_{i}$. For that purpose, we start with a simpler related random variable, which is described in the following lemma.

Lemma 1: For $v \sim \mathcal{U}\left(\mathcal{S}_{t}\right)$ and a fixed $v_{0} \in \mathcal{S}_{t}$, the random variable $\gamma_{0}=\left|\left\langle v, v_{0}\right\rangle\right|^{2}$ has a beta distribution with parameters of 1 and $t-1$. That is, the density function for $\gamma_{0}$ is given by

$$
f_{\gamma_{0}}(x)=(t-1)(1-x)^{t-2}, \quad 0<x<1 .
$$

Similarly, $\xi_{0}:=1-\gamma_{0}$ has a beta distribution with parameters of $t-1$ and 1

$$
f_{\xi_{0}}(x)=(t-1) x^{t-2}, \quad 0<x<1 .
$$

\section{Proof: See Appendix I.}

Now let us look at the conditional density of $\gamma_{i}=\left|\left\langle v, \hat{v}_{i}\right\rangle\right|^{2}$ given $v \in \overline{\mathcal{R}}_{i}$. From the optimum encoding given in (13) or (14), generally each quantization cell has a complicated shape with boundaries defined by neighboring code vectors, and the quantization cells could be all different shapes. This geometrical complexity in the quantization cells makes it difficult to obtain the exact conditional density for $\gamma_{i}$ with a closed-form 


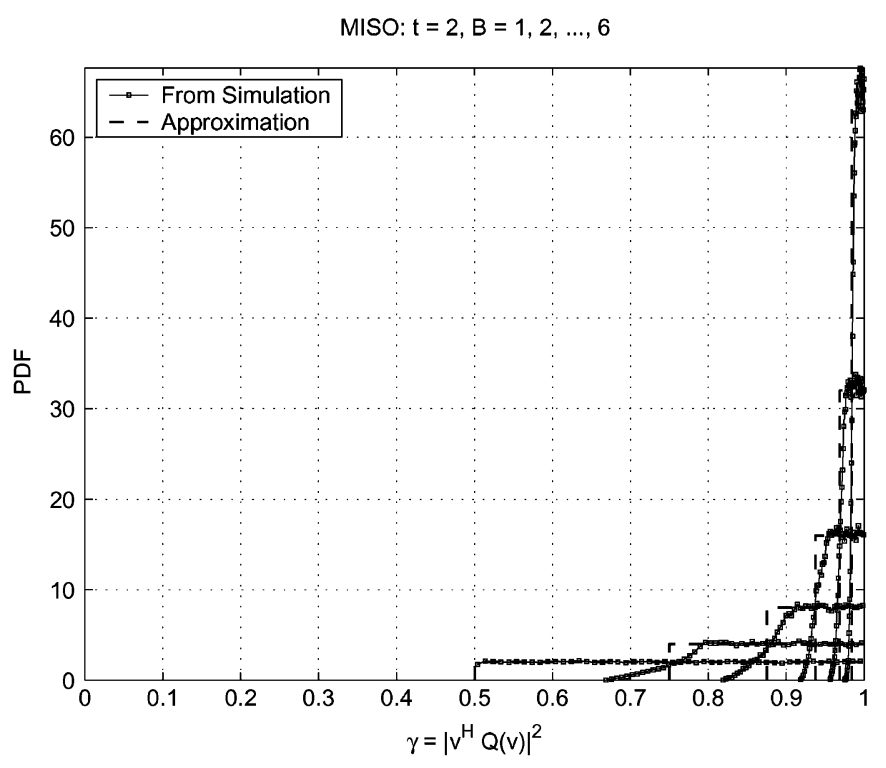

(a)

Fig. 4. Approximate density $\tilde{f}_{\gamma}(\gamma)(B=1,2, \ldots, 6)$. (a) $t=2$, (b) $t=3$.

expression. Using intuitive arguments, we now develop an approximation to this conditional density and in Section IV-B we provide mathematical insight into the nature of the approximation.

When $N$ is reasonably large, since $v$ is uniformly distributed over $\mathcal{S}_{t}, P\left(v \in \overline{\mathcal{R}}_{i}\right) \simeq 1 / N$ for all $i$, and furthermore the shapes of quantization cells will be approximately identical. For analytical tractability, in this paper we constantly consider the following approximation for the quantization cell:

$$
\overline{\mathcal{R}}_{i} \simeq \tilde{\mathcal{R}}_{i}=\left\{v \in \mathcal{S}_{t}: 1-\left|\left\langle v, \hat{v}_{i}\right\rangle\right|^{2} \leq \delta\right\}
$$

for all $i$ and some $\delta>0$, which will be determined as a function of $B$. Under large $N$ assumption, since $v \sim \mathcal{U}\left(\mathcal{S}_{t}\right)$, it is reasonable to assume that $P\left(v \in \overline{\mathcal{R}}_{i}\right)=1 / N$ for all $i$. We solve for $\delta$ to satisfy the condition

$$
P\left(v \in \overline{\mathcal{R}}_{i}\right)=P\left(\gamma_{i} \geq 1-\delta\right)=\int_{1-\delta}^{1} f_{\gamma_{i}}(x) d x=\frac{1}{2^{B}} .
$$

Here note that with the quantization cell approximation of (21), when $v \in \tilde{\mathcal{R}}_{i}, \gamma_{i}$ has the same density as $\gamma_{0}$. That is, $f_{\gamma_{i}}(x)=$ $f_{\gamma_{0}}(x)$ for $1-\delta<x<1$. Then, using the density for $\gamma_{0}$ which was derived in Lemma 1, it can be easily shown that

$$
\delta=2^{-\frac{B}{t-1}} .
$$

Although generally there are overlaps in the approximated quantization cells, the analytical results from the approximation turn out to be quite accurate even when $N$ is small. It should be mentioned that the quantization cell approximation of (21) is similar in some sense to those in quantization error analysis for high-rate VQ in source coding study (for reference see, e.g., [19], [20, Ch. 5]). In the high-rate VQ study, a quantization cell is approximated with the hyper-ellipsoid having the same volume of the cell. In our case, each quantization cell is approximated to the simple geometrical region defined in (21) having

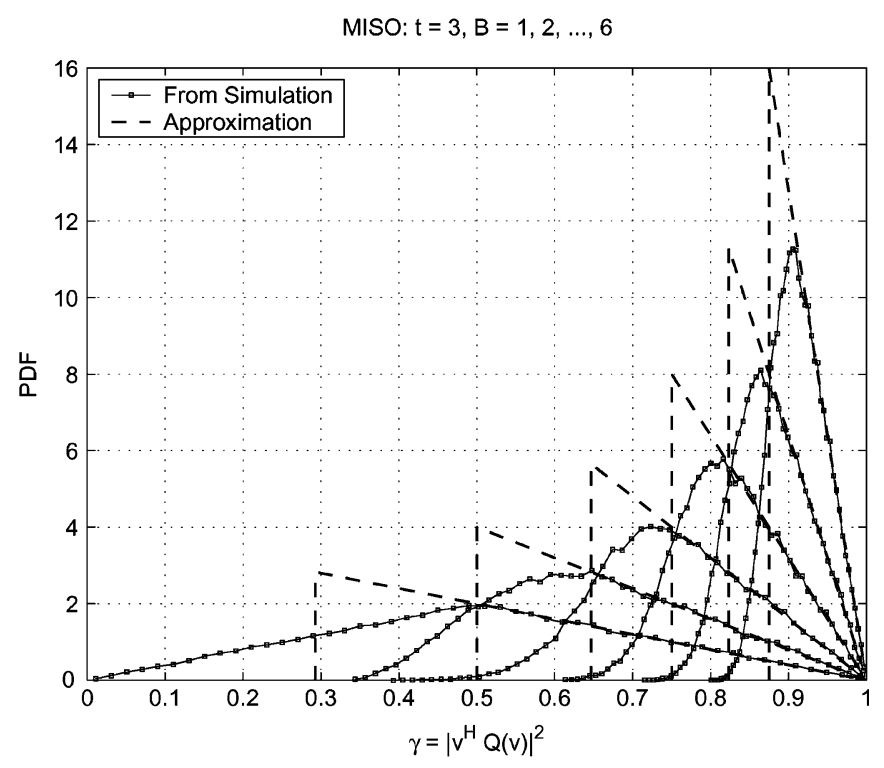

(b)

the same probability as the cell. In [4], Mukkavilli et al. also considered a similar geometrical region (called spherical cap) on a constant-norm sphere to obtain a union bound for the area of no outage region.

With the quantization cell approximation, since the approximated cells $\tilde{\mathcal{R}}_{i}$ have identical geometrical shape and the probabilities $P\left(v \in \tilde{\mathcal{R}}_{i}\right)$ are all equal to $1 / 2^{B}$, the random variables $\gamma_{i}$ given $v \in \tilde{\mathcal{R}}_{i}$ have the same density

$$
\begin{aligned}
f_{\gamma_{i}}\left(x \mid v \in \tilde{\mathcal{R}}_{i}\right) & =\frac{f_{\gamma_{0}}(x) 1_{[1-\delta, 1)}(x)}{P\left(v \in \tilde{\mathcal{R}}_{i}\right)} \\
& =2^{B} f_{\gamma_{0}}(x) 1_{[1-\delta, 1)}(x), \quad \text { for all } i
\end{aligned}
$$

where $1_{A}(x)$ is the indicator function having 1 if $x \in A$ and 0 otherwise. Therefore, we can focus on a particular quantization cell. As a result, we arrive at the following approximation for the density function.

Approximate Density: With the quantization cell approximation described in (21) and (22), the density function for $\gamma=$ $|\langle v, \mathcal{Q}(v)\rangle|^{2}$ is approximated by a truncated beta distribution

$$
f_{\gamma}(x) \simeq \tilde{f}_{\gamma}(x)=2^{B} f_{\gamma_{0}}(x) 1_{[1-\delta, 1)}(x) .
$$

Similarly, $\xi=1-\gamma$ is approximated by

$$
f_{\xi}(x) \simeq \tilde{f}_{\xi}(x)=2^{B} f_{\xi_{0}}(x) 1_{[0, \delta)}(x) .
$$

The above approximate density will be used to derive analytical results for the capacity loss (in Section IV-C), the outage probability (in Section V), and the bit error probability (in Section VI). Fig. 4(a) and (b) shows the approximate densities of $\gamma$ for $t=2$ and 3 and various $B$, together with real densities from simulations using codebooks designed by the MSIP method. We can see that as $B$ increases the distribution of the MSIP value moves toward one. The approximate density functions follow 
this behavior of the simulation results. And if we look at a particular approximate density, it is very close to its counterpart (from simulation) in the area around $\gamma=1$, but they do not agree around $\gamma=\delta$. This is a result of the quantization cell approximation.

\section{B. Performance Bounds Using the Approximate Density Function}

Here we quantify the nature of approximation and show that the quantization cell approximation discussed in Section IV-A leads to a lower bound on the capacity loss. The following lemma is important in proving the main result.

Lemma 2: For $1-\delta \leq x<1, f_{\gamma}(x) \leq \tilde{f}_{\gamma}(x)$.

Proof: See Appendix II.

Theorem 1: The capacity loss $\tilde{C}_{L}$ that is obtained with the approximate density $\tilde{f}_{\gamma}$ is a lower bound on the actual capacity loss $C_{L}$ associated with the true density $f_{\gamma}$. That is

$$
\tilde{C}_{L} \leq C_{L}
$$

Proof: We can write the capacity loss as an expectation over $\gamma$ and $\alpha^{2}$. That is,

$$
\begin{aligned}
C_{L} & =E_{h}\left[I_{L}(h, \hat{v})\right] \\
& =E_{\gamma, \alpha^{2}}\left[I_{L}\left(\alpha^{2}, \gamma\right)\right] \\
& =E_{\gamma}\left\{E_{\alpha^{2} \mid \gamma}\left[I_{L}\left(\alpha^{2}, \gamma\right)\right]\right\}
\end{aligned}
$$

where

$$
I_{L}\left(\alpha^{2}, \gamma\right)=-\log \left(1-\frac{\alpha^{2} P_{T}}{1+\alpha^{2} P_{T}}(1-\gamma)\right) .
$$

Let us define the conditional expectation inside the braces in (26) by $I_{L}(\gamma)$. Note that $I_{L}(\gamma)$ is a monotonically decreasing function in $\gamma$. Then, the theorem can be proved as follows (the integration variable $\gamma$ is omitted for simplicity):

$$
\begin{aligned}
C_{L}= & \int_{0}^{1} I_{L}(\gamma) f_{\gamma}(\gamma) d \gamma \\
= & \int_{1-\delta}^{1} I_{L} \tilde{f}_{\gamma} d \gamma-\int_{1-\delta}^{1} I_{L}\left[\tilde{f}_{\gamma}-f_{\gamma}\right] d \gamma+\int_{0}^{1-\delta} I_{L} f_{\gamma} d \gamma \\
\geq & \int_{1-\delta}^{1} I_{L} \tilde{f}_{\gamma} d \gamma-I_{L}(1-\delta) \int_{1-\delta}^{1}\left[\tilde{f}_{\gamma}-f_{\gamma}\right] d \gamma \\
& +I_{L}(1-\delta) \int_{0}^{1-\delta} f_{\gamma} d \gamma \\
= & \int_{1-\delta}^{1} I_{L} \tilde{f}_{\gamma} d \gamma+I_{L}(1-\delta)\left[\int_{0}^{1} f_{\gamma} d \gamma-\int_{1-\delta}^{1} \tilde{f}_{\gamma} d \gamma\right] \\
= & \int_{1-\delta}^{1} I_{L}(\gamma) \tilde{f}_{\gamma}(\gamma) d \gamma=\tilde{C}_{L}
\end{aligned}
$$

where (27) is because $\tilde{f}_{\gamma}(\gamma)-f_{\gamma}(\gamma) \geq 0$ for $1-\delta \leq \gamma<1$ from Lemma 2 and $I_{L}(\gamma)$ is a monotonically decreasing function in $\gamma$.

Theorem 1 can be generalized to any monotonically decreasing function in $\gamma$. For example, the outage probability and the symbol error probability that are obtained using $\tilde{f}_{\gamma}$ are also lower bounds on the real performances. Similarly, we can show that for any monotonically increasing function in $\gamma$, the approximate density results in an upper bound.

\section{Capacity Loss With Quantized Beamforming}

Now returning to the original problem of capacity loss which we recall can be written as

$$
C_{L}=E\left[-\log \left(1-\frac{\alpha^{2} P_{T}}{1+\alpha^{2} P_{T}} \xi\right)\right]
$$

Using the Taylor series expansion $-\log (1-x)=\sum_{k=1}^{\infty} x^{k} / k$, (28) can be expanded as

$$
C_{L}=\sum_{k=1}^{\infty} \frac{1}{k} E\left[\left(\frac{P_{T} \alpha^{2}}{1+P_{T} \alpha^{2}}\right)^{k}\right] E\left[\xi^{k}\right]
$$

where the independence of $\alpha$ with $v$, hence with $\xi$, is used.

First, using (22) and the approximate density given in (25), we have

$$
\begin{aligned}
E\left[\xi^{k}\right] & =\int_{0}^{\delta} x^{k} 2^{B}(t-1) x^{t-2} d x \\
& =\frac{t-1}{k+t-1} 2^{-\frac{k B}{t-1}} .
\end{aligned}
$$

To calculate the first expectation in (29), the density function for $\alpha^{2}$ is necessary. It is well known that $\alpha^{2}=\|h\|^{2}$ has a gamma distribution [21]

$$
f_{\alpha^{2}}(x)=\frac{x^{t-1} e^{-x}}{\Gamma(t)}, \quad x>0
$$

Using this, the expectation is calculated as follows:

$$
\begin{aligned}
E\left[\left(\frac{P_{T} \alpha^{2}}{1+P_{T} \alpha^{2}}\right)^{k}\right] & =\int_{0}^{\infty}\left(\frac{P_{T} x}{1+P_{T} x}\right)^{k} \frac{x^{t-1} e^{-x}}{\Gamma(t)} d x \\
& =\frac{P_{T}{ }^{k}}{\Gamma(t)} \int_{0}^{\infty} \frac{x^{k+t-1} e^{-x}}{\left(1+P_{T} x\right)^{k}} d x \\
& =\frac{\Gamma(k+t)}{\Gamma(t)} P_{T}{ }^{-t} \Psi\left(k+t, t+1 ; 1 / P_{T}\right) \\
& =\frac{\Gamma(k+t)}{\Gamma(t)} P_{T}{ }^{k}{ }_{2} F_{0}\left(k+t, k ; ;-P_{T}\right)
\end{aligned}
$$

where $\Psi(\alpha, \gamma ; z)$ is the confluent hypergeometric function which has the integral representation [22, eq. (9.211.4)]

$$
\Psi(\alpha, \gamma ; z)=\frac{1}{\Gamma(\alpha)} \int_{0}^{\infty} e^{-z x} x^{\alpha-1}(1+x)^{\gamma-\alpha-1} d x
$$

and ${ }_{2} F_{0}$ is the generalized hypergeometric function [22, eq. (9.14) ${ }_{p} F_{q}$ with $p=2$ and $q=0$. Equation (32) can be easily obtained with a change variable $y=P_{T} x$ and (33) is from the identity [23]

$$
\Psi(\alpha, \beta ; z)=z^{-\alpha} F_{0}(\alpha, \alpha-\beta+1 ; ;-1 / z) .
$$




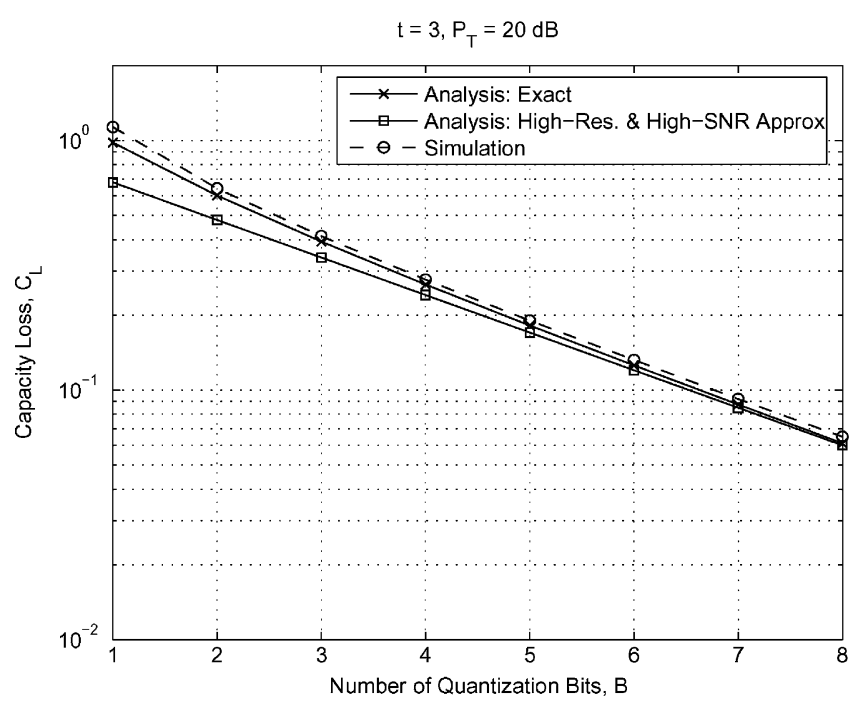

(a)

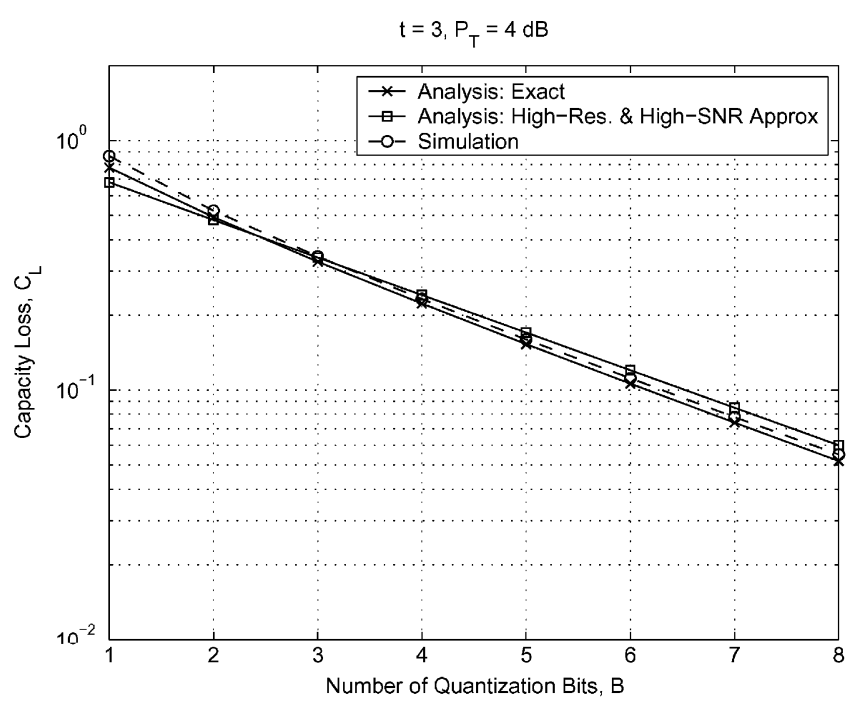

(b)

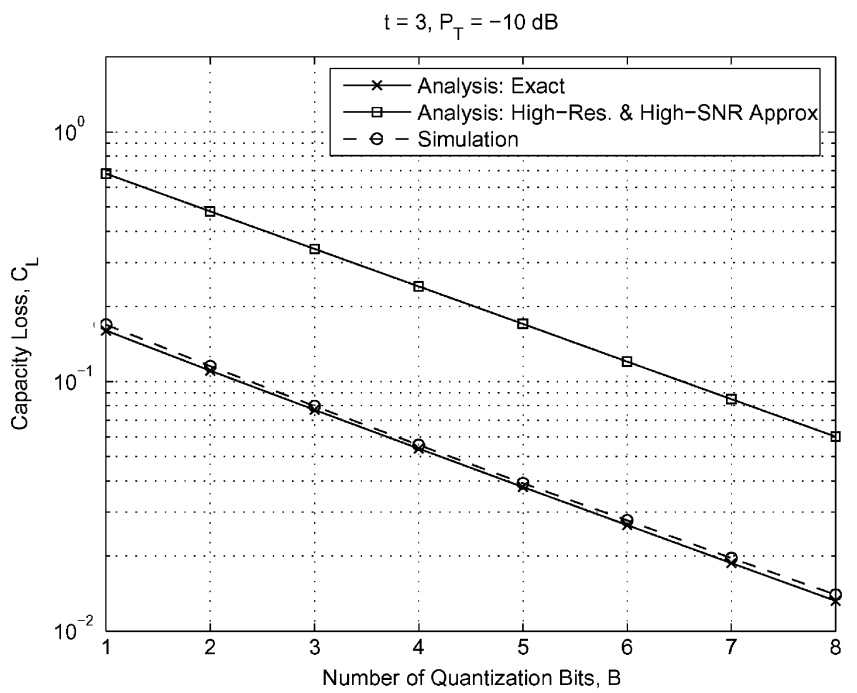

(c)

Fig. 5. Capacity loss due to quantization of beamforming vector $(t=3 ; B=1,2, \ldots, 8)$. Here "Exact" refers to $\tilde{C}_{L}$ given in (34). (a) $P_{T}=20 \mathrm{~dB}$, (b) $P_{T}=$ $4 \mathrm{~dB}$, (c) $P_{T}=-10 \mathrm{~dB}$.

Putting (30) and (33) into (29), finally we have

$$
\tilde{C}_{L}=\frac{t-1}{\Gamma(t)} \sum_{k=1}^{\infty} \frac{\Gamma(k+t)}{k+t-1} 2^{-\frac{k B}{t-1}} P_{T}{ }_{2}{ }_{2} F_{0}\left(k+t, k ; ;-P_{T}\right) .
$$

From the expression in (34), we note that $\tilde{C}_{L}$ goes to zero as $B$ increases to infinity.

1) Approximation to the Capacity Loss: Although the expression in (34) is quite accurate as will be shown later, it is difficult to get insights into how the performance changes in terms of $t$ and $B$. Therefore, in this subsection, an approximation to the capacity loss will be examined. The approximation is derived from $I_{L}(h, \hat{v})$ which is given in (7). When $|\langle v, \hat{v}\rangle| \simeq 1$ and $P_{T} \gg 1$ (called high-resolution and high-SNR assumption), $I_{L}(h, \hat{v})$ can be approximated as follows:

$$
I_{L}(h, \hat{v}) \simeq 1-|\langle v, \hat{v}\rangle|^{2} .
$$

From the SNR expression in (3), we can see that (35) can be also interpreted as the fractional loss in the received SNR with quantized beamforming since the fractional loss is given by

$$
\begin{aligned}
\operatorname{SNR}_{L}(h, \hat{v}) & =\frac{\alpha^{2} P_{T}-\alpha^{2}|\langle v, \hat{v}\rangle|^{2} P_{T}}{\alpha^{2} P_{T}} \\
& =1-|\langle v, \hat{v}\rangle|^{2} .
\end{aligned}
$$

For the approximated capacity loss, we can simply take the expectation of $\xi=1-|\langle v, \hat{v}\rangle|^{2}$ using its approximate density in $(25)$

$$
\begin{aligned}
\tilde{C}_{L} & \simeq 2^{B} \int_{0}^{\delta} x f_{\xi_{0}}(x) d x \\
& =\left(\frac{t-1}{t}\right) 2^{-\frac{B}{t-1}}
\end{aligned}
$$

This is also the average fractional loss in SNR

$$
\mathrm{SNR}_{L}=E_{h}\left[\operatorname{SNR}_{L}(h, \hat{v})\right] \simeq\left(\frac{t-1}{t}\right) 2^{-\frac{B}{t-1}} .
$$




\section{Numerical Results and Discussion}

Fig. 5(a), (b), and (c) shows the capacity loss for MISO systems $(t=3)$ when the beamforming vector is quantized with $B=1, \ldots, 8$, at $P_{T}=20,4$, and $-10 \mathrm{~dB}$, respectively. In each part of the figure, the following three results are plotted in bits per channel use: the exact analytical result $\tilde{C}_{L}$ given in (34), the high-resolution and high-SNR approximation given in (37), and the simulation results using the codebooks designed with the MSIP method. At high SNR, as shown in Fig. 5(a), the results from the approximation as well as the exact one are close to the simulation results. In particular, the exact analytical result $\tilde{C}_{L}$ (which is actually a lower bound as discussed in Section IV-B) and the high-SNR approximation are very tight.

Fig. 5(c) shows the behaviors in low-SNR region. The exact analytical result follows the simulations results, but the approximation deviates from the simulation results with a slope similar to that of the simulation curve. This is as expected because in the approximation, the effect of the term $\alpha^{2} P_{T} /\left(1+\alpha^{2} P_{T}\right)$, which on average is away from 1 in low $P_{T}$, was ignored and assumed to be 1 . Only the dependency of the inner product $\gamma$ with respect to the number of quantization bits was taken into account. As a result, the approximation results in higher values in the capacity loss. This reconfirms that the approximation holds only when the SNR is reasonably high.

The numerical and simulation results for the capacity loss are shown in Fig. 6 for various $t$ and $B$. We can see that more quantization bits are required to meet a certain value of the capacity loss, e.g., $C_{L}=10^{-1}$, as the dimension increases. Also, the slope of the curve decreases as $t$ increases.

Although very accurate results can be obtained using the exact analytical expression given in (34), one can gain insights into the overall behavior through (37)

$$
\tilde{C}_{L} \simeq\left(\frac{t-1}{t}\right) 2^{-\frac{B}{t-1}}
$$

which is a simple function of $t$ and $B$. Similar asymptotic results, though performance metrics are different, can be found in [4 (see eqs. (53) and (54))]. It is interesting to compare (37) with [4, eq. (54)]. The average fractional loss in SNR was approximated as $[4$, eq. (54)]

$$
\mathrm{SNR}_{L} \simeq\left(\frac{t-1}{t}\right) 2^{-\frac{B}{t}}
$$

using asymptotic results in [3, eq. (21)], which was obtained using rate-distortion theory. The two asymptotic expressions are the same except for different factors in $B$. This difference is because in [3], the MSE (Euclidean distance) instead of the MSIP was used when employing rate-distortion theorem for deriving the asymptotic result.

\section{Outage Probability With Quantized FeEdBack}

Another important performance measure for fading channels is the outage probability, especially when the ergodicity requirement $T \gg T_{\text {coh }}$ (meaning that the transmission time is much larger than the channel coherence time) cannot be satisfied [24]. In this section, the outage probability studied in [4] is analyzed

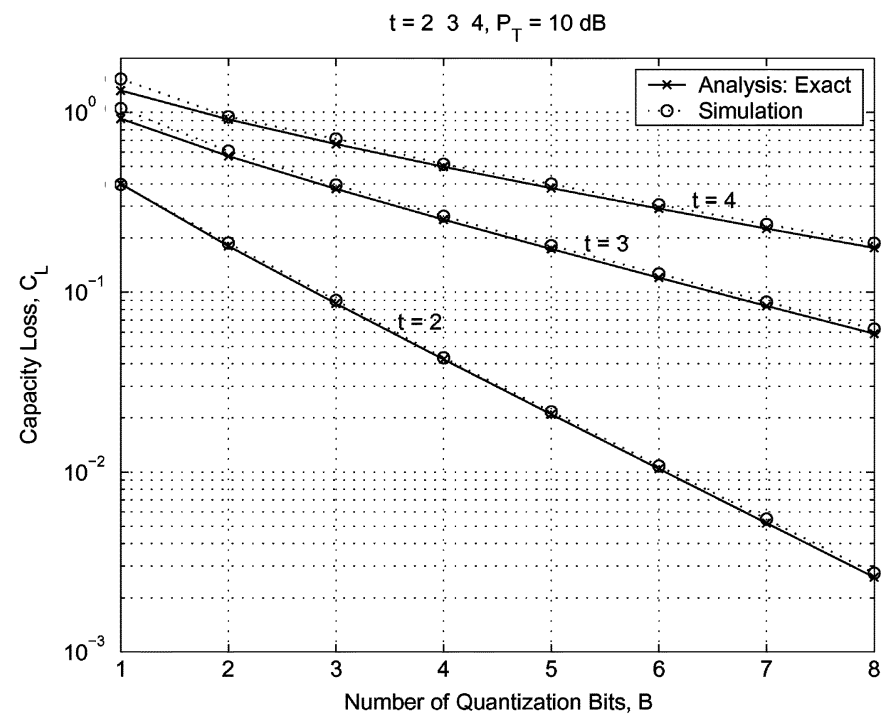

Fig. 6. Capacity loss due to quantization of beamforming vector $\left(t=2,3,4 ; P_{T}=10 \mathrm{~dB} ; B=1,2, \ldots, 8\right)$. Here "Exact" refers to $\tilde{C}_{L}$ given in (34).

by directly applying the distributions derived in Section IV. The outage probability for a given transmit power $P_{T}$ and rate $R$ (bits per channel use) is defined as

$$
P_{\text {out }}\left(R, P_{T}\right)=P[I(h, \hat{v})<R] .
$$

It can be rewritten in terms of $\alpha^{2}$ and $\gamma$ as

$$
\begin{aligned}
P_{\text {out }}\left(R, P_{T}\right) & =P\left(\alpha^{2} \gamma<\beta_{0}\right) \\
& =E_{\alpha^{2}}\left[P\left(\gamma<\frac{\beta_{0}}{\alpha^{2}} \mid \alpha^{2}\right)\right]
\end{aligned}
$$

where $\beta_{0}=\left(2^{R}-1\right) / P_{T}$.

Using the approximate density $\tilde{f}_{\gamma}$ in (24), the conditional probability in (38) is given by

$$
\begin{aligned}
& P\left(\gamma<\frac{\beta_{0}}{\alpha^{2}} \mid \alpha^{2}\right) \\
& \quad \simeq \begin{cases}1, & \alpha^{2} \leq \beta_{0} \\
2^{B}\left[\delta^{t-1}-\left(1-\frac{\beta_{0}}{\alpha^{2}}\right)^{t-1}\right], & \beta_{0}<\alpha^{2}<\beta_{1} \\
0, & \alpha^{2} \geq \beta_{1}\end{cases}
\end{aligned}
$$

where $\beta_{1}=\beta_{0} /(1-\delta)$. Then, by averaging (39) with respect to $f_{\alpha^{2}}$ given in (31), we have a lower bound ${ }^{7}$ on the outage probability

$$
\begin{aligned}
P_{\text {out }} & \left(R, P_{T}\right) \\
\geq & \int_{0}^{\beta_{0}} f_{\alpha^{2}}(x) d x \\
& +\int_{\beta_{0}}^{\beta_{1}} 2^{B}\left[\delta^{t-1}-\left(1-\frac{\beta_{0}}{\alpha^{2}}\right)^{t-1}\right] f_{\alpha^{2}}(x) d x \\
= & \int_{0}^{\beta_{1}} f_{\alpha^{2}}(x) d x-2^{B} \int_{\beta_{0}}^{\beta_{1}}\left(1-\frac{\beta_{0}}{\alpha^{2}}\right)^{t-1} f_{\alpha^{2}}(x) d x
\end{aligned}
$$

${ }^{7}$ This claim of lower bound comes from Theorem 1. 


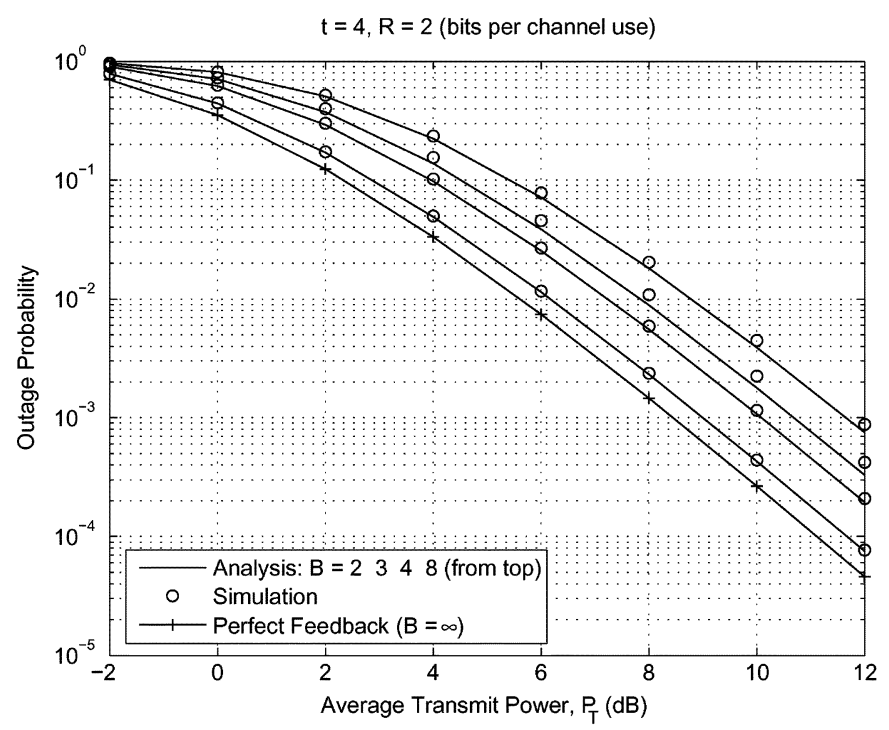

Fig. 7. Outage probability of MISO channels with quantized beamforming $(t=4 ; R=2$ bits per channel use; $B=2,3,4,8)$.

where we used $2^{B} \delta^{t-1}=1$. We define the cumulative distribution function of $\alpha^{2}$ as $F(t, \beta)$ which is given by [21]

$$
\begin{aligned}
F(t, \beta) & =\int_{0}^{\beta} f_{\alpha^{2}}(x) d x \\
& =1-e^{\beta} \sum_{k=0}^{t-1} \frac{\beta^{k}}{k !} .
\end{aligned}
$$

The second integral in (40) can be expressed in the form of $e^{-\beta_{0}} F\left(t, \beta_{1}-\beta_{0}\right)$, resulting in

$$
P_{\text {out }}\left(R, P_{T}\right) \geq F\left(t, \beta_{1}\right)-2^{B} e^{-\beta_{0}} F\left(t, \beta_{1}-\beta_{0}\right) .
$$

By using (41), finally we arrive at

$$
P_{\text {out }}\left(R, P_{T}\right) \geq 1-2^{B} e^{-\beta_{0}}+e^{-\beta_{1}} \sum_{k=0}^{t-1} \frac{2^{B}\left(\beta_{1}-\beta_{0}\right)^{k}-\beta_{1}^{k}}{k !} \text {. }
$$

Interestingly, this bound is exactly the same as the one derived in [4] and our analysis provide an alternate derivation.

Fig. 7 shows the numerical results from (43) for the outage probability (when $t=4, R=2$ bits per channel use and various $B$ ), together with simulation results using codebook designed by the MSIP method. As can be seen, there is pretty good agreement between the analytical results and the simulations.

\section{Bit ERRor Probability With QuANTIZEd FeEdBack}

Although the capacity loss and the outage probability discussed in the previous sections are good performance measures for ideally channel-coded systems, the bit error probability is also useful in practice. In this section, the effect of quantization of the beamforming vector on the bit error probability will be analyzed. Consider a uncoded MISO system with transmit beamforming along $\hat{v}$. The received signal is represented as

$$
\begin{aligned}
y & =\alpha|\langle v, \hat{v}\rangle| s+\eta \\
& =c s+\eta
\end{aligned}
$$

where $\eta \sim \tilde{\mathcal{N}}\left(0, \sigma_{\eta}^{2}\right)$ and $c=\alpha|\langle v, \hat{v}\rangle|$ is the composite channel gain between the transmit symbol $s$ and the received signal $y$. In this section, we present results for the case of binary signaling $s= \pm \sqrt{P_{T}}$ and coherent detection at the receiver. However, we would like to note that the approach introduced in this section is general and not limited to the binary case. The decision statistics is given as

$$
z=\operatorname{Re}\left[c^{*} y\right]=|c|^{2} s+\eta^{\prime}
$$

where $\eta^{\prime}=\operatorname{Re}\left[c^{*} \eta\right] \sim \mathcal{N}\left(0, \sigma_{\eta}^{2} / 2\right)$.

The probability of bit error for a given channel $h$ is

$$
\begin{aligned}
P(E \mid h) & =P\left(z<0 \mid s=\sqrt{P_{T}}\right) \\
& =Q\left(\sqrt{\frac{2|c|^{2} P_{T}}{\sigma_{\eta}^{2}}}\right) \\
& =Q\left(\sqrt{2 \alpha^{2} \gamma \Gamma}\right)
\end{aligned}
$$

where $E$ is the error event, $Q(x)$ is the $Q$-function defined as

$$
Q(x)=\frac{1}{\sqrt{2 \pi}} \int_{x}^{\infty} e^{-t^{2} / 2} d t
$$

$\gamma=|\langle v, \hat{v}\rangle|^{2}$ as before, and $\Gamma=P_{T} / \sigma_{\eta}^{2}$ (transmit SNR). Taking an expectation over channel realizations and noting that two random variables $\alpha^{2}$ and $\gamma$ are independent, the bit error probability is given by

$$
\begin{aligned}
P(E) & =E_{h}[P(E \mid h)] \\
& =E_{\alpha^{2}, \gamma}\left[P\left(E \mid \alpha^{2}, \gamma\right)\right] \\
& =E_{\gamma}\left[E_{\alpha^{2} \mid \gamma}\left[P\left(E \mid \alpha^{2}, \gamma\right)\right]\right] \\
& =\int_{0}^{1}\left[\int_{0}^{\infty} Q\left(\sqrt{2 \alpha^{2} \gamma \Gamma}\right) f_{\alpha^{2}}\left(\alpha^{2}\right) d \alpha^{2}\right] f_{\gamma}(\gamma) d \gamma .
\end{aligned}
$$

First, we consider the conditional probability of error for a given $\gamma$ denoted by $P(E \mid \gamma)$, which is the integral inside the bracket in (45). Since $\alpha^{2}$ has the gamma distribution given by (31)

$$
\begin{aligned}
P(E \mid \gamma) & =E_{\alpha^{2} \mid \gamma}\left[P\left(E \mid \alpha^{2}, \gamma\right)\right] \\
& =\int_{0}^{\infty} Q\left(\sqrt{2 \alpha^{2} \gamma \Gamma}\right) f_{\alpha^{2}}\left(\alpha^{2}\right) d \alpha^{2} \\
& =\frac{1}{\Gamma(t)} \int_{0}^{\infty} Q\left(\sqrt{2 \alpha^{2} \gamma \Gamma}\right) \alpha^{2(t-1)} e^{-\alpha^{2}} d \alpha^{2} \\
& =\left(\frac{1-\mu(\gamma \Gamma)}{2}\right) \sum_{k=0}^{t-1}\left(\begin{array}{c}
t-1+k \\
k
\end{array}\right)\left(\frac{1+\mu(\gamma \Gamma)}{2}\right)^{k}
\end{aligned}
$$

where

$$
\mu(a)=\sqrt{\frac{a}{1+a}}
$$

and the last step is well known in the maximal ratio combining (MRC) and the maximal ratio transmission (MRT) analysis, e.g., [25], [26]. By taking an average over $\gamma$ using the density $\tilde{f}_{\gamma}$ derived in (24), we obtain a lower bound on the bit error probability

$$
\begin{aligned}
\tilde{P}(E) & =\frac{2^{B}(t-1)}{2^{t}} \sum_{k=0}^{t-1}\left(\begin{array}{c}
t-1+k \\
k
\end{array}\right) \frac{1}{2^{k}} \\
& \cdot \int_{1-\delta}^{1}(1-\mu(\gamma \Gamma))^{t}(1+\mu(\gamma \Gamma))^{k}(1-\gamma)^{t-2} d \gamma
\end{aligned}
$$




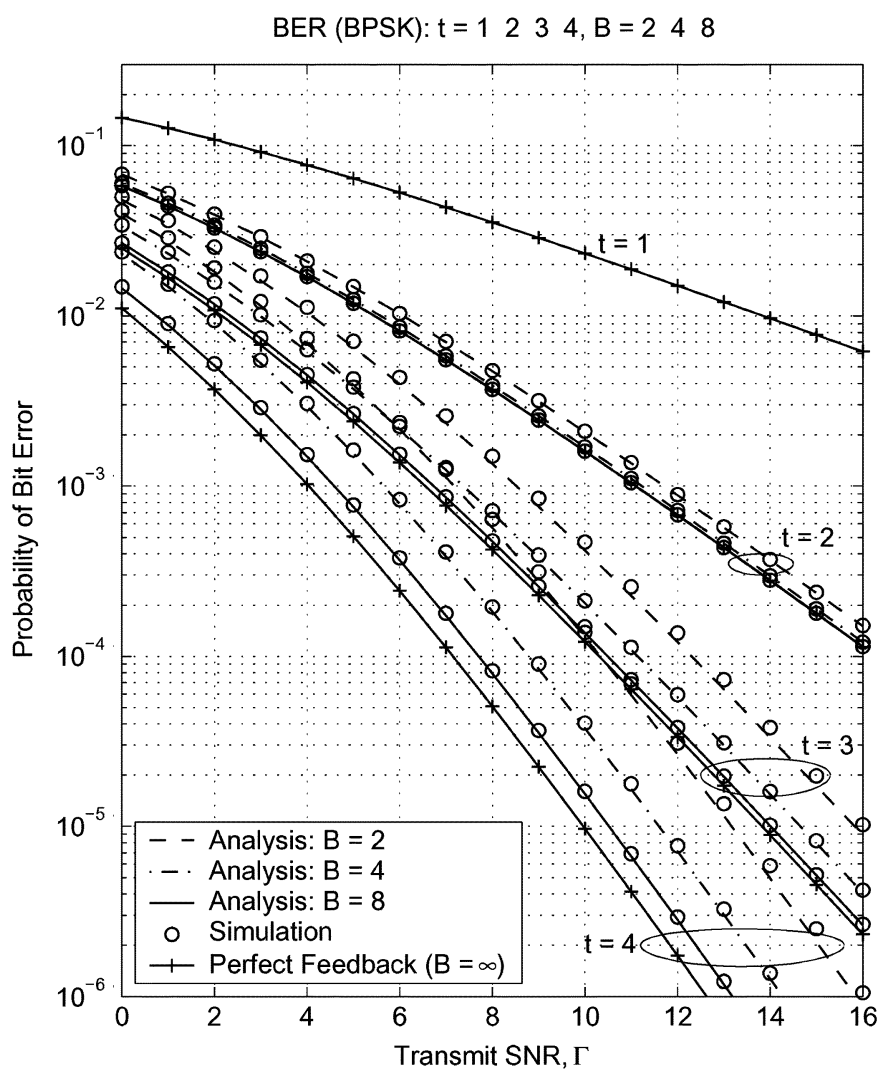

Fig. 8. Bit error probability of MISO systems with quantized beamforming $(t=1,2,3,4 ; B=2,4,8)$.

where $\delta=2^{-\frac{B}{t-1}}$. This can be calculated numerically without difficulty.

Fig. 8 shows the numerical and the simulation results for the bit error probability with varying number of transmit antennas $(t=1,2,3,4)$, and quantization bits $(B=2,4,8)$. The results for the perfect feedback, i.e., $B=\infty$, are also plotted for comparison. We can see that, as the dimension increases, more quantization bits are required to get a close performance to the perfect feedback. Similar behavior was also observed in the previous section for the capacity loss analysis. The lower bound appears to be very tight based on the simulation results. A similar approach for the symbol error probability analysis based on the geometrical framework presented in [4] can be found in [27].

\section{CONCLUSION}

We have investigated the problem of quantization associated with beamforming in multiple-antenna communication systems with finite-rate feedback. We proposed a new quantizer design criterion, namely, MSwIP, and developed a Lloyd-type quantizer design algorithm. With the MSwIP quantizer design method, we can design the optimum beamforming codebook for an arbitrary number of transmit antennas and quantization bits, and for any spatial correlation structure in channel. To analyze the performance of MISO systems with quantized beamforming, the i.i.d. Rayleigh-fading case was considered and the density function of the squared inner product between the optimum and the quantized beamforming vector was derived by considering an approximation of the quantization cell. Then, the density function was used to analyze the capacity loss due to quantization. An approximation to the capacity loss was also derived to gain insights into the performance behavior. The approximation has a simple analytical expression in terms of the number of transmit antennas and the number of quantization bits. The power of the approach was further demonstrated by applying the derived density function to analyze the outage probability and the bit error probability for a finite-rate feedback MISO system.

\section{APPENDIX I \\ PROOF OF LEMMA 1}

From assumptions, $h \sim \tilde{\mathcal{N}}\left(0, I_{t}\right)$ and $v_{0}$ is a fixed vector in $\mathcal{S}_{t}$. Let us decompose $h$ into $v_{0}$ and its orthogonal complements $v_{0}^{\perp} \in \mathbb{C}^{t \times(t-1)}$

$$
h=h_{a} v_{0}+v_{0}^{\perp} h_{b}
$$

where $h_{a}$ is a scalar and $h_{b} \in \mathbb{C}^{t-1}$. It is easily shown that $h_{a}=v_{0}^{\dagger} h$ and $h_{b}=\left(v_{0}^{\perp}\right)^{\dagger} h$, and that $\left|h_{a}\right|^{2} \sim \Gamma(1,1),\left\|h_{b}\right\|^{2} \sim$ $\Gamma(t-1,1)$ and they are independent, where $\Gamma(p, \lambda)$ means the gamma distribution with parameters $(p, \lambda)$. Since $\|h\|^{2}=$ $\left|h_{a}\right|^{2}+\left\|h_{b}\right\|^{2}, \gamma_{0}$ can be written as

$$
\gamma_{0}=\left|\left\langle v, v_{0}\right\rangle\right|^{2}=\frac{\left|v_{0}^{\dagger} h\right|^{2}}{\|h\|^{2}}=\frac{\left|h_{a}\right|^{2}}{\left|h_{a}\right|^{2}+\left\|h_{b}\right\|^{2}} .
$$

It is a known fact that, if $X_{1}$ and $X_{2}$ are independent random variables with $\Gamma(p, \lambda)$ and $\Gamma(q, \lambda)$, respectively, then $Z=X_{1} /\left(X_{1}+X_{2}\right)$ has $\beta(p, q)$, the beta distribution with parameters $(p, q)$ (see, e.g., [28, Theorem 1.2.3]). By applying this, we arrive at the desired result $\gamma_{0} \sim \beta(1, t-1)$. Similarly, since $\xi_{0}=\left\|h_{b}\right\|^{2} /\left(\left|h_{a}\right|^{2}+\left\|h_{b}\right\|^{2}\right), \xi_{0} \sim \beta(t-1,1)$.

\section{APPENDIX II \\ PROOF OF LEMMA 2}

Let us define

$$
\begin{aligned}
& \overline{\mathcal{R}}_{i, x}=\left\{v \in \mathcal{S}_{t}: v \in \overline{\mathcal{R}}_{i} \text { and }\left|\left\langle v, \hat{v}_{i}\right\rangle\right|^{2} \geq x\right\} \\
& \tilde{\mathcal{R}}_{i, x}=\left\{v \in \mathcal{S}_{t}:\left|\left\langle v, \hat{v}_{i}\right\rangle\right|^{2} \geq x\right\} .
\end{aligned}
$$

Note that the above sets have the following properties: i) $\overline{\mathcal{R}}_{i, x} \subseteq$ $\tilde{\mathcal{R}}_{i, x}$ since $\overline{\mathcal{R}}_{i, x}=\tilde{\mathcal{R}}_{i, x} \cap \overline{\mathcal{R}}_{i}$. ii) $\overline{\mathcal{R}}_{i, x} \supseteq \overline{\mathcal{R}}_{i, x+\Delta x}$ and also $\tilde{\mathcal{R}}_{i, x} \supseteq \tilde{\mathcal{R}}_{i, x+\Delta x}$ for a small positive $\Delta x$. iii) $P\left(\overline{\mathcal{R}}_{i, x}\right) / P\left(\overline{\mathcal{R}}_{i}\right)=$ $1-F_{\gamma}(x)$, similarly, $P\left(\tilde{\mathcal{R}}_{i, x}\right) / P\left(\tilde{\mathcal{R}}_{i}\right)=1-\tilde{F}_{\gamma}(x)$, where $P(A)$ means $P(v \in A)$, and $F_{\gamma}$ and $\tilde{F}_{\gamma}$ are the cumulative distribution functions corresponding to $f_{\gamma}$ and $\tilde{f}_{\gamma}$, respectively.

We claim that

$$
\overline{\mathcal{R}}_{i, x} \backslash \overline{\mathcal{R}}_{i, x+\Delta x} \subseteq \tilde{\mathcal{R}}_{i, x} \backslash \tilde{\mathcal{R}}_{i, x+\Delta x}
$$

where $A \backslash B=A \cap B^{c}$ is the set difference of $A$ and $B$. This claim can be proved as follows:

$$
\begin{aligned}
\overline{\mathcal{R}}_{i, x} \backslash \overline{\mathcal{R}}_{i, x+\Delta x} & =\left(\tilde{\mathcal{R}}_{i, x} \backslash \tilde{\mathcal{R}}_{i, x+\Delta x}\right) \cap \overline{\mathcal{R}}_{i} \\
& \subseteq \tilde{\mathcal{R}}_{i, x} \backslash \tilde{\mathcal{R}}_{i, x+\Delta x}
\end{aligned}
$$


where we used property i) and an identity $(A \cap C) \backslash(B \cap C)=$ $(A \backslash B) \cap C$. From (48), we have

$$
P\left(\overline{\mathcal{R}}_{i, x} \backslash \overline{\mathcal{R}}_{i, x+\Delta x}\right) \leq P\left(\tilde{\mathcal{R}}_{i, x} \backslash \tilde{\mathcal{R}}_{i, x+\Delta x}\right) .
$$

Using property ii), it can be written as

$$
P\left(\overline{\mathcal{R}}_{i, x}\right)-P\left(\overline{\mathcal{R}}_{i, x+\Delta x}\right) \leq P\left(\tilde{\mathcal{R}}_{i, x}\right)-P\left(\tilde{\mathcal{R}}_{i, x+\Delta x}\right) .
$$

Remembering that $P\left(\overline{\mathcal{R}}_{i}\right) \simeq 1 / N$ and $P\left(\tilde{\mathcal{R}}_{i}\right) \simeq 1 / N$

$$
\frac{P\left(\overline{\mathcal{R}}_{i, x}\right)}{P\left(\overline{\mathcal{R}}_{i}\right)}-\frac{P\left(\overline{\mathcal{R}}_{i, x+\Delta x}\right)}{P\left(\overline{\mathcal{R}}_{i}\right)} \leq \frac{P\left(\tilde{\mathcal{R}}_{i, x}\right)}{P\left(\tilde{\mathcal{R}}_{i}\right)}-\frac{P\left(\tilde{\mathcal{R}}_{i, x+\Delta x}\right)}{P\left(\tilde{\mathcal{R}}_{i}\right)}
$$

By using property iii) and dividing both sides by $\Delta x$, we have

$$
\frac{F_{\gamma}(x+\Delta x)-F_{\gamma}(x)}{\Delta x} \leq \frac{\tilde{F}_{\gamma}(x+\Delta x)-\tilde{F}_{\gamma}(x)}{\Delta x} .
$$

Finally, taking a limit $\Delta x \rightarrow 0$, we arrive at $f_{\gamma}(x) \leq \tilde{f}_{\gamma}(x)$.

\section{REFERENCES}

[1] İ. E. Telatar, "Capacity of multi-antenna Gaussian channels," Europ. Trans. Telecommun., vol. 10, no. 6, pp. 585-595, Nov./Dec. 1999.

[2] E. Biglieri, G. Caire, and G. Taricco, "Limiting performance of blockfading channels with multiple antennas," IEEE Trans. Inf. Theory, vol. 47, no. 4, pp. 1273-1289, May 2001.

[3] A. Narula, M. J. Lopez, M. D. Trott, and G. W. Wornell, "Efficient use of side information in multiple-antenna data transmission over fading channels," IEEE J. Sel. Areas Commun., vol. 16, pp. 1423-1436, Oct. 1998.

[4] K. K. Mukkavilli, A. Sabharwal, E. Erkip, and B. Aazhang, "On beamforming with finite rate feedback in multiple antenna systems," IEEE Trans. Inf. Theory, vol. 49, no. 10, pp. 2562-2579, Oct. 2003.

[5] F. Rashid-Farrokhi, K. J. R. Liu, and L. Tassiulas, "Transmit beamforming and power control for cellualr wireless systems," IEEE J. Sel. Areas Commun., vol. 16, no. 8, pp. 1437-1450, Oct. 1998.

[6] R. T. Derryberry et al., "Transmit diversity in 3G CDMA systems," IEEE Commun. Mag., vol. 40, pp. 68-75, Apr. 2002.

[7] D. J. Love, R. Heath Jr., and T. Strohmer, "Grassmannian beamforming for multiple-input multiple-output wireless systems," IEEE Trans. Inf. Theory, vol. 49, no. 10, pp. 2735-2747, Oct. 2003.

[8] W. Santipach and M. L. Honig, "Asymptotic performance of MIMO wireless channels with limited feedback," in Proc. IEEE MILCOM 2003, Boston, MA, Oct. 2003, pp. 141-146.

[9] — , "Asymptotic capacity of beamforming with limited feedback," in Proc. IEEE Int. Symp. Information Theory, Chicago, IL, Jun./Jul. 2004, p. 290.
[10] A. Gersho and R. M. Gray, Vector Quantization and Signal Compression. Norwell, MA: Kluwer Academic, 1992.

[11] R. M. Gray and D. L. Neuhoff, "Quantization," IEEE Trans. Inf. Theory, vol. 44, no. 6, pp. 2325-2383, Oct. 1998.

[12] Y. Linde, A. Buzo, and R. M. Gray, "An algorithm for vector quantizer design," IEEE Trans. Commun., vol. COM-28, no. 1, pp. 84-95, Jan. 1980.

[13] V. K. N. Lau, Y. Liu, and T. A. Chen, "On the design of MIMO blockfading channels with feedback-link capacity constraint," IEEE Trans. Commun., vol. 52, no. 1, pp. 62-70, Jan. 2004.

[14] J. C. Roh and B. D. Rao, "Design and analysis of MIMO spatial multiplexing systems with quantized feedback," IEEE Trans. Signal Process., to be published.

[15] — - "MIMO spatial multiplexing systems with limited feedback," in Proc. IEEE Int. Conf. Communications, Seoul, Korea, May 2005, pp. $777-782$.

[16] P. Xia and G. B. Giannakis, "Design and analysis of transmit-beamforming based on limited-rate feedback," in Proc. IEEE Vehicular Technology Conf., Los Angeles, CA, Sep. 2004, pp. 1653-1657.

[17] D. J. Love and R. Heath Jr., "Grassmannian beamforming on correlated MIMO channels," in Proc. IEEE GLOBECOM 2004, Dollas, TX, Dec. 2004, pp. 106-110.

[18] J. Salz and J. H. Winters, "Effect of fading correlation on adaptive arrays in digital mobile radio," IEEE Trans. Veh. Technol., vol. 43, no. 4, pp. 1049-1057, Nov. 1994.

[19] Y. Yamada, S. Tazaki, and R. M. Gray, "Asymptotic performance of block quantizers with difference distortion measures," IEEE Trans. Inf. Theory, vol. IT-26, no. 1, pp. 6-14, Jan. 1980.

[20] R. M. Gray, Source Coding Theory. Norwell, MA: Kluwer Academic, 1990.

[21] J. G. Proakis, Digital Communications, 3rd ed: McGraw-Hill, 1995.

[22] I. S. Gradshteyn and I. M. Ryzhik, Table of Integrals, Series and Products, 5th ed. San Diego, CA: Academic Press, 1994.

[23] A. Erdelyi, Higher Transcendental Functions Vol. I. New York: McGraw-Hill, 1953.

[24] E. Biglieri, J. Proakis, and S. Shamai (Shitz), "Fading channels: Information theoretic and communications aspects," IEEE Trans. Inf. Theory, vol. 44, no. 6, pp. 2619-2692, Oct. 1998.

[25] M. K. Simon and M.-S. Alouini, Digital Communication Over Fading Channels: A Unified Approach to Performance Analysis. New York: Wiley, 2000.

[26] J. K. Cavers, "Single-user and multiuser adaptive maximal ratio transmission for Rayleigh channels," IEEE Trans. Veh. Technol., vol. 49, no. 6, pp. 2043-2050, Nov. 2000.

[27] S. Zhou, Z. Wang, and G. B. Giannakis, "Performance analysis of transmit-beamforming with finite-rate feedback," in Proc. 38th Conf. Information Sciences and Systems. Princeton, NJ, Mar. 2004, pp. 880-885.

[28] P. J. Bickel and K. A. Doksum, Mathematical Statistics: Basic Ideas and Selected Topics. Englewood Cliffs, NJ: Prentice-Hall, 1977. 